\title{
Noether Symmetries and Critical Exponents
}

\author{
Yuri BOZHKOV
}

Departamento de Matemática Aplicada - DMA, Instituto de Matemática, Estatistica e Computação Científica - IMECC, Universidade Estadual de Campinas UNICAMP, C.P. 6065, 13083-970 - Campinas - SP, Brasil

E-mail: bozhkov@ime.unicamp.br

URL: http://www.ime.unicamp.br/ bozhkov/

Received October 03, 2005, in final form November 19, 2005; Published online November 25, 2005

Original article is available at http://www.emis.de/journals/SIGMA/2005/Paper022/

\begin{abstract}
We show that all Lie point symmetries of various classes of nonlinear differential equations involving critical nonlinearities are variational/divergence symmetries.
\end{abstract}

Key words: divergence symmetry; critical exponents

2000 Mathematics Subject Classification: 34C14; 35A15

\section{Introduction}

The main purpose of this paper is to discuss a common property of certain classes of quasilinear differential equations, namely: a Lie point symmetry of the considered equation is a Noether symmetry if and only if the equation parameters assume critical values.

By 'Noether symmetry' we mean a variational or a divergence symmetry. Further, as it is well known, the so-called critical exponent is found as the critical power for embedding theorems. It is also related to some numbers dividing the existence and nonexistence cases for the solutions of differential equations, in particular, of semilinear differential equations with power nonlinearities involving the Laplace operator. The above stated property traces a connection between these two notions: the Noether symmetries and the 'criticality' of the equation.

We shall review the results obtained in $[4,5,6,3]$, where that property was established for various differential equations. To the author's knowledge, the 'enigmatic' relation between the Noether symmetries and the critical exponents has not previously been emphasized.

Our research was inspired by the paper of Clement, de Figueiredo and Mitidieri [7] in which these authors introduced the following class of quasilinear ordinary differential equations:

$$
-\left(x^{\alpha}\left|y^{\prime}\right|^{\beta} y^{\prime}\right)^{\prime}=x^{\gamma} f(y),
$$

where $\alpha, \beta$ and $\gamma$ are real numbers, $x>0, y=y(x)$ and $f$ is a non-negative function. The relations between these parameters will be specified below. We point out that for specific values of $\alpha, \beta, \gamma$ it contains well-known important differential equations briefly listed in Section 2 . In particular, it contains the radial forms of partial differential equations involving Laplace or $p$-Laplace operators. Our aim is to investigate the cases, in terms of the equation parameters, for which the stated in the beginning property holds.

We call the equation (1) the model equation. It is is considered for $x \in(0, R), 0<R \leq \infty$, with the conditions

$$
y^{\prime}(0)=0, \quad y(R)=0,
$$

and one looks for positive solutions. 
We are interested in nonlinearities of power or exponential type. According to [7] we distinguish the following two cases:

- The Sobolev case: $f(y)=\lambda y^{p}, \lambda=$ const, and

$$
\alpha-\beta-1>0
$$

- The Pohozaev-Trudinger case: $f(y)=\lambda e^{y}, \lambda=$ const, and

$$
\alpha=\beta+1 \text {. }
$$

If the equations involved the Laplace operator, both cases are related with the exceptional cases of the Sobolev theorem. In this regard we recall that the existence of solution to the equation $\Delta u+u^{p}=0$ depends essentially on the number $p$ characterizing the nonlinearity. Namely, it admits positive solutions if and only if $p \geq 2^{*}=(n+2) /(n-2)$ - the critical Sobolev exponent [9].

For the Sobolev case the critical exponent associated to (1) was found in [7]. Its value is given by

$$
q^{*}=\frac{(\gamma+1)(\beta+2)}{\alpha-\beta-1} .
$$

Some explicit formulas for the solutions corresponding to the above cases were found in [7], among other results. The relations (3) and (4) were essentially used in that work. It is natural to raise the following questions:

What is the relationship between the conditions (3) and (4) and the nature of the equation (1)? In what context do they appear? What is the role of the critical exponent? After all, why was it possible to find the exact solutions?

We asked and answered these questions in [4, 5]. In those works the Lie point symmetry group of the model equation is calculated. Then it is verified which of the found symmetries are Noether symmetries. In this way, the property mentioned in the beginning regarding Noether symmetries and critical exponents, is established on the base of the S. Lie Theory. Since in the most cases the proof of embedding theorems between function spaces is reduced to radially symmetric functions, an ordinary differential equation is obtained. The symmetry approach permits as well the critical exponent be defined using directly that ordinary differential equation, without involving functional analysis. Namely: it is the only exponent for which a Lie point symmetry of (1) with $f(y)=\lambda y^{p}$ is a variational symmetry.

Further, in [6] and [3] respectively, similar problems are considered for systems of ordinary differential equations and scalar partial differential equations. In this paper we shall present the main results of $[4,5,6,3]$. Our exposition in some parts follows closely the text of the original articles. It corresponds to the talk we gave on June 22, 2005, during the 6th International Conference 'Symmetry in Nonlinear Mathematical Physics', June 20-26 2005, Kyiv, Ukraine.

\section{The model equation}

The model equation contains the following particular cases:

1. The equation (1) is the radial form of partial differential equations containing

- the Laplace operator in $\mathbb{R}^{N}$ if $\alpha=\gamma=N-1, \beta=0$;

- the $p$-Laplace operator in $\mathbb{R}^{N}$ if $\alpha=\gamma=N-1, \beta=p-2$;

- the $k$-Hessian operator in $\mathbb{R}^{N}$ if $\alpha=N-k, \gamma=N-1, \beta=k-1$. 
2. The equation (1) with $\alpha=\gamma=N-1, \beta=0$ and $f(y)=\lambda e^{y}$ arises in the Liouville-Gelfand problem.

3. The generalized Lane-Emden equation of the first kind:

$$
y^{\prime \prime}+\frac{\tilde{\alpha}}{x} y^{\prime}+\tilde{\beta} x^{\nu-1} y^{n}=0,
$$

can be obtained from (1) by setting $\beta=0, \alpha=\tilde{\alpha}, f(y)=\tilde{\beta} y^{n}$, and $\gamma=\alpha+\nu-1$. The equation (6) with $\tilde{\alpha}=2$ is the Emden-Fowler equation, and if, in addition, $\nu=1$, then (6) is the equation proposed by Lane and studied in detail by Emden.

This equation and its particular cases appear in astrophysics, mechanics, general relativity, various theories of gravitation, atomic physics and quantum mechanics.

4. The generalized Lane-Emden equation of the second kind:

$$
y^{\prime \prime}+\frac{\tilde{\alpha}}{x} y^{\prime}+\tilde{\beta} x^{\nu-1} e^{n y}=0 .
$$

5. The Boltzmann equation

$$
-\left(x y^{\prime}\right)^{\prime}=x \sum_{i=1}^{m} \lambda_{i} k_{i} e^{-k_{i} y},
$$

which is used in a huge number of applications, in particular, in the biophysics study of DNA modelled as a spiral on a cylinder .

\section{The Sobolev case for the model equation}

In this section we consider the model equation (1) with $f(y)=\lambda y^{p}, \lambda>0$ and $\beta>-1$, which can be written in the following form:

$$
y^{\prime \prime}=-\frac{\alpha}{\beta+1} \frac{y^{\prime}}{x}-\frac{\lambda}{\beta+1} x^{\gamma-\alpha}\left|y^{\prime}\right|^{-\beta} y^{p} .
$$

The symmetry group of (7) is calculated in [5], where various cases, depending on some relations between the parameters, are considered. Although that may be interesting from the point of view of the group analysis, we shall not present the complete results here directing the interested reader to [5] for further details. Rather, in order to establish the property stated in the introduction regarding Noether symmetries and critical exponents, we shall concentrate on the case when

$$
\alpha-\beta-1>0
$$

and

$$
\beta+1<p \leq q^{*}-1
$$

where the critical exponent $q^{*}$ is given by (5). In this way, for the sake of clarity and brevity, we shall avoid some considerations which do not contribute to clarifying of that property. Moreover, these conditions are natural, since they are valid in the most of the interesting particular cases of the model equation. The following theorem is a direct corollary of the results obtained in [5].

Theorem 1. Let (8) and (9) hold. Then the Lie point symmetry group of (7) is generated by the vector field

$$
X_{1}=\frac{\beta+1-p}{\gamma-\alpha+\beta+2} x \frac{\partial}{\partial x}+y \frac{\partial}{\partial y} .
$$


Proof. By (8) and(9) it follows that $\gamma-\alpha+\beta+2>0$. (The latter condition is necessary for the existence of a positive solution of our problem [7].) It is also easy to see that (8) and (9) imply $\alpha+\gamma+\beta \gamma \neq 0$. Then the theorem follows from the results in [5].

Further we observe that the equation (7) is the Euler-Lagrange equation of the functional

$$
J[y]=\int_{0}^{R} L\left(x, y, y^{\prime}\right) d x
$$

where the function of Lagrange $L=L\left(x, y, y^{\prime}\right)$ is given by

$$
L\left(x, y, y^{\prime}\right)=\frac{1}{\beta+2} x^{\alpha}\left|y^{\prime}\right|^{\beta+2}-\frac{\lambda}{p+1} x^{\gamma} y^{p+1} .
$$

Our next purpose is to check which of the symmetries leave the Euler functional $J[y]$ invariant, that is, which of the already found in [5] Lie point symmetries are actually variational. Again we consider only the case in the Theorem 1 in which all symmetries form a one-parameter group generated by $X_{1}$.

Theorem 2 ([5]). A Lie point symmetry of the equation (7) is a variational symmetry if and only if $p+1$ is equal to the critical exponent.

Proof. We denote

$$
k=\frac{\beta+1-p}{\gamma-\alpha+\beta+2} .
$$

The Lie point transformation corresponding to (10) is given by

$$
\begin{aligned}
& x^{*}=\mu^{k} x, \\
& y^{*}=\mu y .
\end{aligned}
$$

Clearly this is a scaling transformation. Then

$$
\begin{aligned}
J\left[y^{*}\right] & =\frac{1}{\beta+2} \int_{0}^{R^{*}}\left(x^{*}\right)^{\alpha}\left|\frac{d y^{*}}{d x^{*}}\left(x^{*}\right)\right|^{\beta+2} d x^{*}-\frac{\lambda}{p+1} \int_{0}^{R^{*}}\left(x^{*}\right)^{\gamma}\left(y^{*}\left(x^{*}\right)\right)^{p+1} d x^{*} \\
& =\frac{\mu^{k(\alpha-\beta-1)+\beta+2}}{\beta+2} \int_{0}^{R} x^{\alpha}\left|y^{\prime}\right|^{\beta+2} d x-\frac{\lambda \mu^{k(\gamma+1)+p+1}}{p+1} \int_{0}^{R} x^{\gamma} y^{p+1} d x .
\end{aligned}
$$

Hence $J\left[y^{*}\right]=J[y]$ if and only if both exponents of $\mu$ vanish:

$$
\begin{aligned}
& k(\alpha-\beta-1)+\beta+2=0, \\
& k(\gamma+1)+p+1=0 .
\end{aligned}
$$

The latter two equalities hold if and only if $p=q^{*}-1$.

Henceforth, to the end of this section, we shall suppose $p=q^{*}-1$. Then by the Noether theorem we obtain that

$$
\frac{\beta+1}{\alpha-\beta-1} x^{\alpha+1}\left|y^{\prime}\right|^{\beta+2}-x^{\alpha} y\left|y^{\prime}\right|^{\beta+1}+\frac{\lambda}{\gamma+1} x^{\gamma+1} y^{q^{*}}=0,
$$

whenever $y$ is a solution of the equation (7).

Taking a look at [7], one can observe that the above first integral, up to some constant multiplier, is the $\psi$ used in [7, p. 154]. Further we express $\lambda x^{\gamma-\alpha} y^{q^{*}-1}$ from (11) as a function 
of $x$ and $y^{\prime}$ and then substitute it into the equation (7). We thus obtain that $y^{\prime}$ must satisfy the following equation

$$
y^{\prime \prime}=\frac{(\gamma-\alpha+1)}{\beta+1} \frac{1}{x} y^{\prime}+\frac{\gamma+1}{\alpha-\beta-1} \frac{1}{y} y^{\prime 2},
$$

whose solution, represented below, can be easily found by setting $y^{\prime}=v y$. In this way we have come to the following theorem of [7]:

Theorem 3 ([7]). The problem

$$
\begin{aligned}
& -\left(x^{\alpha}\left|y^{\prime}\right|^{\beta} y^{\prime}\right)^{\prime}=\lambda x^{\gamma} y^{q^{*}-1} \quad \text { in } \quad(0, \infty), \\
& y(0)=y_{0}>0, \quad y^{\prime}(0)=0 \text {, } \\
& y>0 \quad \text { in }[0, \infty) \text {, }
\end{aligned}
$$

has the unique solution

$$
y_{*}(x)=\left(y_{0}{ }^{-\sigma}+k_{0} x^{s}\right)^{-1 / \sigma},
$$

where

$$
\sigma=\frac{\gamma-\alpha+\beta+2}{\alpha-\beta-1}, \quad s=\frac{\gamma-\alpha+\beta+2}{\beta+1} \quad \text { and } \quad k_{0}=\frac{\beta+1}{\alpha-\beta-1}\left(\frac{\lambda y_{0}{ }^{\sigma}}{\gamma+1}\right)^{1 /(\beta+1)} .
$$

The reason for the success in solving (12) is fact that the exponent in the right-hand side of the equation is critical, and hence any Lie point symmetry is variational, which reduces the order of integration procedure by two. In particular, this explains why the Lane-Emden equation

$$
y^{\prime \prime}+\frac{2}{x} y^{\prime}+y^{5}=0
$$

can be explicitly solved. Indeed, if the spatial dimension $n=3$, then the corresponding equation parameters are $2=n-1$ and $5=(n+2) /(n-2)$ - the Sobolev exponent.

\section{The Pohozaev-Trudinger case for the model equation}

Denote by $B$ the sphere in $\mathbb{R}^{N}$ with centre at the origin and radius $R$. Then the Liouville-Gelfand problem consists of finding positive solutions of the following equation

$$
-\Delta u=\lambda e^{u} \quad \text { in } B
$$

with the Dirichlet boundary condition:

$$
u=0 \quad \text { on } \quad \partial B .
$$

The equation above has been proposed and studied by Liouville. He found an exact solution in dimension $N=1$, and also (for $N=2$ ) a solution in terms of an arbitrary harmonic function. Bratu [2] found two explicit solutions of the problem if $0<\lambda<2 / R^{2}$ and $N=2$. Gelfand [8] considered, among other things, the problem of thermal self-ignition of a chemically active mixture of gases in plane, cylindrical and spherical vessels. For $N=3$ he investigated the values of the parameter $\lambda$ for which the problem has a solution and studied the multiplicity of such solutions.

It can be proved that if $\lambda \leq 0$ or if $\lambda$ is greater than a certain positive constant $\lambda^{*}$ there is no solution of the boundary value problem (13), (14). Then by the celebrated results of Gidas, Ni 
and Nirenberg [10] it follows that if a solution exists, then it must be radially symmetric. Thus the Liouville-Gelfand problem is reduced to the study of the following problem for an ordinary differential equation:

$$
\begin{array}{ll}
-y^{\prime \prime}-\frac{N-1}{x} y^{\prime}=\lambda e^{y} & \text { in } \quad(0, R), \\
y^{\prime}(0)=y(R)=0, & \text { in } \quad[0, R) . \\
y>0 &
\end{array}
$$

In [7] Clement, de Figueiredo and Mitidieri proposed the following generalization :

$$
\begin{array}{ll}
-\left(x^{\alpha}\left|y^{\prime}\right|^{\beta} y^{\prime}\right)^{\prime}=\lambda x^{\gamma} e^{y} & \text { in } \quad(0, R), \\
y^{\prime}(0)=y(R)=0, & \text { in }[0, R), \\
y>0 & {[0,}
\end{array}
$$

assuming that

$$
\alpha-\beta-1=0, \quad \beta>-1, \quad \gamma>-1,
$$

that is, the situation corresponds to the Pohozaev-Trudinger case.

Clement, de Figueiredo and Mitidieri found in [7] the above mentioned constant $\lambda^{*}$, proved that there is only one solution of (15) if $\lambda=\lambda^{*}$, and that there exist exactly two solutions if $0<\lambda<\lambda^{*}$. Moreover, using the method of first integrals, they found the explicit formulas for these solutions. Again the reason for the success of the integration is the fact that the condition $\alpha=\beta+1$ used in [7] holds if and only if all Lie point symmetries are variational symmetries, and hence the order of the integration procedure reduces by two. This is proved in [4] where it is shown that the exact solutions can be accounted for by symmetry techniques. To see this, one needs first to find the Lie point symmetries of the model equation with $f(y)=\lambda e^{u}, \lambda>0$. We present the result in the following reduced form

Theorem 4 ([4]). Suppose that $\beta>-1, \alpha-\beta-1 \geq 0$ and $\gamma-\alpha+\beta+2>0$. If $\beta \neq 0$ or if $\beta=0, \alpha \neq 1$, the Lie point symmetry group of the equation

$$
-\left(x^{\alpha}\left|y^{\prime}\right|^{\beta} y^{\prime}\right)^{\prime}=\lambda x^{\gamma} e^{y}
$$

is generated by

$$
X_{2}=-\frac{1}{m} x \frac{\partial}{\partial x}+\frac{\partial}{\partial y}
$$

where $m=\gamma-\alpha+\beta+2$. If $\beta=0$ and $\alpha=1$ the symmetry group is the two-parameter Lie group determined by $X_{2}$ and

$$
\frac{1}{\gamma+1}\left(\frac{2}{\gamma+1} x-x \ln x\right) \frac{\partial}{\partial x}+\ln x \frac{\partial}{\partial y} .
$$

In the quasilinear case, it is easy to see by Theorem 4 that the following result holds.

Theorem 5 ([4]). Let $\beta \neq 0$. Then the Lie point symmetry group of

$$
-\left(x^{\alpha}\left|y^{\prime}\right|^{\beta} y^{\prime}\right)^{\prime}=\lambda x^{\gamma} e^{y}
$$

is a variational symmetry group if and only if $\alpha=\beta+1$. 
Further, observe that by Theorem 4, the Lie point symmetry group for $\beta=0$ and $\alpha=1$ is two-dimensional. Hence, one can deduce that in this case there exists a one-dimensional subgroup consisting of variational symmetries.

Then using $\alpha=\beta+1$ and $\left|y^{\prime}\right|=-y^{\prime}$, we obtain the following first integral

$$
\psi\left(x, y, y^{\prime}\right):=\alpha x^{\alpha+1}\left|y^{\prime}\right|^{\alpha+1}-(\alpha+1)(\gamma+1) x^{\alpha}\left|y^{\prime}\right|^{\alpha}+\lambda(\alpha+1) x^{\gamma+1} e^{y}=0 .
$$

Again, taking a look at [7], one can observe that the function $\psi$ above is the same that appears in [7]. Further we express $y$ from (16) as a function of $x$ and $y^{\prime}$ and then substitute it into the equation in (15). We obtain that $y^{\prime}$ must satisfy the following Bernoulli equation

$$
y^{\prime \prime}=\frac{(\gamma-\alpha+1)}{\alpha} \frac{1}{x} y^{\prime}+\frac{1}{\alpha+1} y^{\prime 2}
$$

which can be easily solved. Thus

$$
y(x)=-(\alpha+1) \ln \left|c_{1}-\frac{\alpha}{(\alpha+1)(\gamma+1)} x^{(\gamma+1) / \alpha}\right|+c_{2} .
$$

By the boundary condition $y(R)=0$ (see $(2)$ ) we obtain that

$$
c_{1}=-\frac{(\gamma+1)^{\alpha}}{\mu_{i}\left(\lambda \mu_{i}\right)^{1 / \alpha}} \quad \text { and } \quad c_{2}=(\alpha+1) \ln \frac{(\gamma+1)^{\alpha}}{\left(\lambda \mu_{i}\right)^{1 / \alpha}}
$$

where $\mu_{i}, i=1,2$, is a root of

$$
H(\mu):=m \mu^{(\alpha+1) / \alpha}-\mu+1=0, \quad m=\frac{\alpha \lambda^{1 / \alpha} R^{(\gamma+1) / \alpha}}{(\alpha+1)(\gamma+1)^{(\alpha+1) / \alpha}} .
$$

In this way we have come to another result of [7]:

Theorem 6 ([7]). The solution(s) of (15) can be represented in the following way:

1) if $0<\lambda<\lambda^{*}:=\frac{(\gamma+1)^{\alpha+1}}{(\alpha+1) R^{\gamma+1}}$ then there are two solutions

$$
y_{1,2}(x)=-(\alpha+1) \ln \left(\frac{1}{\mu_{1,2}}+\frac{\alpha\left(\lambda \mu_{1,2}\right)^{1 / \alpha}}{(\alpha+1)(\gamma+1)^{(\alpha+1) / \alpha}} x^{(\gamma+1) / \alpha}\right)
$$

2) if $\lambda=\lambda^{*}$ then there exists the unique solution

$$
y_{0}(x)=-(\alpha+1) \ln \left(\frac{1}{\mu_{0}}+\frac{\alpha \mu_{0}^{1 / \alpha}}{(\alpha+1)^{(\alpha+1) / \alpha} R^{(\gamma+1) / \alpha}} x^{(\gamma+1) / \alpha}\right),
$$

where $\mu_{0}$ is the unique solution of $H(\mu)=0$;

3) if $\lambda \leq 0$ or $\lambda>\lambda^{*}$ there is no solution.

We observe that we have obtained the solutions found in [7] just by applying symmetry and variational methods, and without use of another first integral

$$
\varphi:=x^{(\sigma-\gamma-1) / \alpha} \frac{d}{d x}\left(e^{-y(x) /(\alpha+1)}\right) .
$$

See [7]. In fact, this $\varphi$ corresponds to a dynamical symmetry. 


\section{The variational symmetries of the Lane-Emden systems}

In Sections 3 and 4, following [4, 5], we have described the properties of the large class of quasilinear ordinary differential equations (1) with power or exponential nonlinearities from the point of view of the Lie symmetry theory. We have shown that the Lie point symmetries of equations which contain critical exponents are actually variational symmetries. In this section we shall present the results of [6] which show that this property is also valid for systems.

The system of two semilinear partial differential equations

$$
\begin{aligned}
& -\Delta u=v^{q}, \\
& -\Delta v=u^{p},
\end{aligned}
$$

where the independent variable $x \in \mathbb{R}^{n}, n \geq 3$ - integer, $p$ and $q$ - positive real numbers, is called the Lane-Emden system. It can be considered as a natural extension of the celebrated Lane-Emden equation

$$
\Delta \theta+\theta^{p}=0
$$

in $\mathbb{R}^{n}$.

We are interested in radial ground state solutions of (17), whose study is reduced to the study of the following system of two ordinary differential equations:

$$
\begin{aligned}
& \ddot{u}+\frac{n-1}{t} \dot{u}+v^{q}=0, \\
& \ddot{v}+\frac{n-1}{t} \dot{v}+u^{p}=0,
\end{aligned}
$$

where $t=|x|, \dot{u}=\frac{d u}{d t}$, etc., and $\dot{u}(0)=\dot{v}(0)=\lim _{t \rightarrow \infty} u(t)=\lim _{t \rightarrow \infty} v(t)=0$.

It is well known that the Lane-Emden equation (18) admits positive solutions if and only if $p$ is greater than or equal to the Sobolev exponent $(n+2) /(n-2)$ (see, for instance, [9]). In [20] J. Serrin and H. Zou prove an analogous result for the system (17). Namely: if $(p, q)$ is on or above the curve in the first quadrant in the $(p, q)$ plane given by

$$
\frac{n}{p+1}+\frac{n}{q+1}=n-2
$$

then there exist infinitely many (componentwise) positive radial solutions $(u, v)$ of (17) tending to $(0,0)$ when $|x| \rightarrow \infty$. That is, the solutions are ground states. This theorem combined with the earlier nonexistence results of E. Mitidieri [13, 15] and J. Serrin, H. Zou [18, 19] gives a complete picture of existence and nonexistence of positive radial solutions of (17) in which the dividing curve (20) plays an important role (see [20, Corollary 1.2, p. 370]). For this reason (20) is called the critical hyperbola.

In regard to existence of ground states for the Lane-Emden system (17), the results of P.-L. Lions [12] and J. Hulshof, R. van der Vorst [11] imply that the problem with $p$ and $q$ satisfying (20) has a unique, up to scalings and translations, ground state, which is positive, radially symmetric and decreasing in the radial component $t=|x|$. We shall come back to this point later.

The basic assumptions on the parameters are:

$$
n \geq 3, \quad p q>1, \quad p \neq q, \quad p \neq 1, \quad q \neq 1 .
$$

Then the symmetry group of the Lane-Emden system (19) is calculated in [6], and we state it in the form of the following 
Theorem 7 ([6]). Suppose that the conditions (21) hold. Then the Lie point symmetry group of the Lane-Emden system (19) is the one-parameter group generated by the vector field

$$
X_{3}=t \frac{\partial}{\partial t}+\frac{2(1+q)}{1-p q} u \frac{\partial}{\partial u}+\frac{2(1+p)}{1-p q} v \frac{\partial}{\partial v} .
$$

Further, following the same argument as in the proof of Theorem 2, we obtain the next

Theorem 8 ([6]). Let the parameters $n, p$ and $q$ satisfy (21). Then any Lie point symmetry of the Lane-Emden system (19) is a variational symmetry if and only if

$$
\frac{n}{2}=\frac{(p+1)(q+1)}{p q-1},
$$

that is, the point $(p, q)$ is on the critical hyperbola (20).

The statement of Theorem 8 is a conjecture of Enzo Mitidieri [16].

We observe that if $(p, q)$ belongs to the critical hyperbola, the Noether Theorem immediately gives the first integral used in [11] and called invariant paraboloid.

We conclude this section noting that the exact solution of the problem (19) is not available. As mentioned above, it is known to exist, and only the asymptotic behavior of such ground state is known. Challenged by Enzo Mitidieri and provoked by J. Hulshof, R. van der Vorst's paper we proposed in [6] the following

Problem. Find the solution of the Lane-Emden system explicitly.

\section{The Lie point symmetries of semilinear polyharmonic equations}

In the next section we shall consider the semilinear polyharmonic equation

$$
(-1)^{k} \Delta^{k} u=f(u)
$$

where $\Delta$ is the Laplace operator in $\mathbb{R}^{n}, n \geq 2$ and $k \geq 1$. The Lie point symmetries of (22) were studied by Svirshchevskii, [22, 23] who obtained the complete group classification. We shall cite partially his result in a form suitable for our purpose to investigate the cases of power and exponential nonlinearities.

Theorem 9 ([22, 23]). The widest Lie point symmetry group admitted by (22) with general $f(u)$ is determined by translations and rotations.

For some special choices of the right-hand side $f(u)$ it can be expanded by additional operators as follows:

If $f(u)=u^{p}, p \neq 0, p \neq 1$, we have the generator of dilations

$$
Z=x_{i} \frac{\partial}{\partial x_{i}}+\frac{2 k}{1-p} u \frac{\partial}{\partial u}
$$

and, for $p=(n+2 k) /(n-2 k)$, there are $n$ additional generators

$$
Y_{i}=\left(2 x_{i} x_{j}-|x|^{2} \delta_{i j}\right) \frac{\partial}{\partial x_{j}}+(2 k-n) x_{i} u \frac{\partial}{\partial u} .
$$

Further, if $f(u)=e^{u}$ then the operator

$$
W=x_{i} \frac{\partial}{\partial x_{i}}-2 k \frac{\partial}{\partial u}
$$


generates a sub-group of the Lie point symmetry group of (22). If $n=2 k$, there are $n$ additional generators

$$
V_{i}=\left(2 x_{i} x_{j}-|x|^{2} \delta_{i j}\right) \frac{\partial}{\partial x_{j}}-4 k x_{i} \frac{\partial}{\partial u} .
$$

Above $\delta_{i j}$ is the Kronecker symbol, $|x|=\left(\sum_{s=1}^{n} x_{s}^{2}\right)^{1 / 2}$ and summation over a repeated index is assumed.

\section{The Noether symmetries of semilinear polyharmonic equations with critical nonlinearities}

Up to this point, we have established the property relating the critical exponents and the Noether symmetries for some ordinary differential equations and systems. It is natural to conjecture that the Lie point symmetries of more general differential equations involving critical exponents are Noether symmetries. In [3] we show that this is valid for a class of partially differential equations. Namely, for the semilinear polyharmonic equation (22).

Again, we shall distinguish two cases: we have

$$
f(u)=u^{p}, \quad n>2 k \geq 2, \quad p \neq 0, \quad p \neq 1,
$$

in the Sobolev case, while in the Pohozaev-Trudinger case

$$
f(u)=e^{u}, \quad n=2 k, \quad k \geq 2 .
$$

Having at our disposal the already cited group classification (see the preceding section), we investigate which of the Lie point symmetries in these cases are variational or divergence symmetries.

The first results in [3] can be formulated as follows:

Theorem 10 ([3]). Suppose that the conditions (23) hold. Then the Lie point symmetry $Z$ (see Theorem 9) of the polyharmonic equation

$$
(-1)^{k} \Delta^{k} u=u^{p}
$$

is a variational symmetry if and only if

$$
p=\frac{n+2 k}{n-2 k},
$$

that is, $p$ is equal to the well known critical exponent.

Theorem 11 ([3]). Let $n \geq 3$ and $k \geq 1$. Then the Lie point symmetry $W$ (see Theorem 9 ) of the polyharmonic equation

$$
(-1)^{k} \Delta^{k} u=e^{u} .
$$

is a variational symmetry if and only if

$$
n=2 k \text {. }
$$


The importance of the relation $n=2 k$ for the polyharmonic equation (1) was pointed out to us by Enzo Mitidieri in January 1995 [14].

The proofs of Theorems 10 and 11 are analogous to the proof of Theorem 2. In fact, this is a dimensional analysis argument since the latter is equivalent to the invariance under scaling transformations (see, for example, $[1,17]$ ).

Since the translations and the rotations are variational symmetries of the considered polyharmonic equation, it follows from Theorems 10 and 11 that in the critical cases the corresponding Lie point symmetry groups contain a subgroup consisting of variational symmetries. However, in the critical cases one can get more.

The main results in [3] are the following

Theorem 12 ([3]). Suppose that the conditions (23) hold. Then any Lie point symmetry of the polyharmonic equation

$$
(-1)^{k} \Delta^{k} u=u^{(n+2 k) /(n-2 k)}
$$

is a divergence symmetry.

Theorem 13 ([3]). Let $n \geq 4, k \geq 2$ and $n=2 k$. Then any Lie point symmetry of the polyharmonic equation

$$
(-1)^{k} \Delta^{k} u=e^{u}
$$

is a divergence symmetry.

The proofs of Theorems 12 and 13 are reduced to the proof of the facts that $Y_{i}$ and $V_{i}$ (see Theorem 9) are divergence symmetries, since the other infinitesimal generators of the corresponding Lie point symmetry groups determine variational symmetries by Theorems 10 and 11 as already observed. For this purpose we find explicitly the vector-valued 'potential' function $B$ determining $Y_{i}$ and $V_{i}$ as divergence symmetries [3].

The importance of the variational and the divergence symmetries is due to the fact that they determine conservation laws via the Noether Theorem $[1,17]$. Thus the next step in this research is to establish the conservation laws corresponding to the already studied variational and divergence symmetries. This is possible since we already have at our disposal the explicit formula for $B$. This is also done in [3].

The Theorems 10-13 confirm the validity of the general property relating Noether symmetries and the critical parameters, stated in the beginning of the introduction. Another examples which illustrate this property are given in [21], in which Svirshchevskii proved that the symmetries of the $p$-Laplace equation

$$
-\operatorname{div}\left(|\nabla u|^{p-2} \nabla u\right)=|u|^{q-1} u
$$

and the equation

$$
-\Delta\left(|\Delta u|^{\sigma} \Delta u\right)=|u|^{q-1} u
$$

are variational if and only if the parameters assume critical values.

\section{Acknowledgements}

We wish to thank the Organizers of the 6th International Conference 'Symmetry in Nonlinear Mathematical Physics', June 20-26 2005, Kyiv, Ukraine, for having given us the opportunity to talk on this subject as well as for their warm hospitality. We are grateful to the referees for their useful suggestions. We would also like to thank FAEPEX-UNICAMP for partial financial support. 
[1] Bluman G.W., Kumei S., Symmetries and differential equations, New York, Springer, 1989.

[2] Bratu G., Sur les équationes intégrales non linéares, Bull. Soc. Math. de France, 1914, V.42, $113-142$.

[3] Bozhkov Y.D., Divergence symmetries of semilinear polyharmonic equations involving critical nonlinearities, submitted.

[4] Bozhkov Y.D., Gilli Martins A.C., On the symmetry group of a differential equation and the LiouvilleGelfand problem, Rend. Istit. Mat. Univ. Trieste, 2002, V.34, 103-120.

[5] Bozhkov Y.D., Gilli Martins A.C., Lie point symmetries and exact solutions of quasilinear differential equations with critical exponents, Nonlinear Anal., 2004, V.57, N 5-6, 773-793.

[6] Bozhkov Y.D., Gilli Martins A.C., Lie point symmetries of the Lane-Emden system, J. Math. Anal. Appl., V.294, 2004, 334-344.

[7] Clément P., de Figueiredo D.G. , Mitidieri E., Quasilinear elliptic equations with critical exponents, Topol. Methods Nonlinear Anal., 1996, V.7, 133-164.

[8] Gelfand I.M., Some problems in the theory of quasilinear equations, Amer. Math. Soc. Transl., 1963, V.29, 295-381.

[9] Gidas B., Spruck J., Global and local behaviour of positive solutions of nonlinear elliptic equations, Comm. Pure Appl. Math., 1981, V.34, 525-598.

[10] Gidas B., Ni W., Nirenberg L., Symmetry and related problems via maximum principle, Comm. Math. Phys., 1979, V.68, 209-243.

[11] Hulshof J., van der Vorst R.C.A.M., Assymptotic behavior of ground states, Proc. Amer. Math. Soc., 1996, V.124, 2423-2431.

[12] Lions P.L., The concentration-compactness principle in the calculus of variations, part 1, Rev. Mat. Iberoam., 1985, V.1, 145-201.

[13] Mitidieri E., A Relich type identity and applications, Commun. Partial Differential Equations, 1993, V.18, 125-151.

[14] Mitidieri E., Private communication, January 1995.

[15] Mitidieri E., Nonexistence of positive solutions of semilinear elliptic systems in $\mathbf{R}^{N}$, Differential Integral Equations, 1996, V.9, 465-479.

[16] Mitidieri E., Private communication, January 2002.

[17] Olver P.J., Applications of Lie groups to differential equations, New York, Springer, 1986.

[18] Serrin J., Zou H., Non-existence of positive solutions of semilinear elliptic systems, Discourses in Mathematics and its Applications, Vol. 3, Department of Mathematics, Texas A\&M University, College Station, Texas, 1994, 55-68.

[19] Serrin J., Zou H., Non-existence of positive solutions of the Lane-Emden systems, Differential Integral Equations, 1996, V.9, 635-653.

[20] Serrin J., Zou H., Existence of positive solutions of the Lane-Emden systems, Atti Sem. Mat. Fis. Univ. Modena, 1998, V.46, suppl., 369-380.

[21] Svirshchevskii S.R., Symmetry of nonlinear elliptic equations with critical values of parameters, Preprint 118, Moscow, Keldysh Institute of Applied Mathematics, Academy of Sciences USSR, 1989.

[22] Svirshchevskii S.R., Symmetries of nonlinear polyharmonic equations, Preprint 11, Moscow, Institute of Mathematical Modelling, Academy of Sciences USSR, 1993.

[23] Svirshchevskii S.R., Group classification of nonlinear polyharmonic equations and their invariant solutions, Diff. Equations, 1993, V.29, 1538-1547 (in Russian: Diferentsial'nye Uravneniya, 1993, V.29, N 10, 17721781). 